\title{
Computer aided cytological cancer diagnosis: Cell type classification as a step towards fully automatic cancer diagnostics on cytopathological specimens of serous effusions
}

Timna E. Schneider and André A. Bell and Dietrich Meyer-Ebrecht and Alfred Böcking and Til Aach

Institute of Imaging and Computer Vision RWTH Aachen University, 52056 Aachen, Germany tel: +49 24180 27860, fax: +49 2418022200

web: www.lfb.rwth-aachen.de

in: SPIE Medical Imaging 2007, Computer-Aided Diagnosis. See also BiBT $\mathrm{E}_{\mathrm{X}} \mathrm{X}$ entry below.

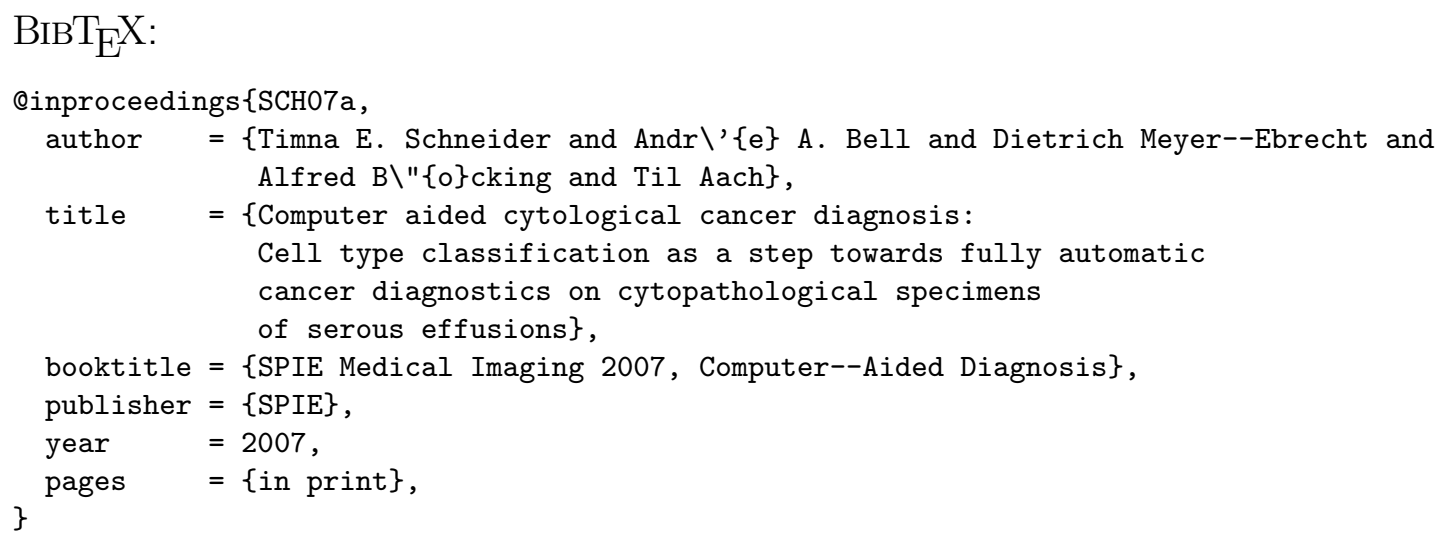

(C) 2007 Society of Photo-Optical Instrumentation Engineers. This paper was published in SPIE Medical Imaging 2007, Computer-Aided Diagnosis and is made available as an electronic reprint with permission of SPIE. One print or electronic copy may be made for personal use only. Systematic or multiple reproduction, distribution to multiple locations via electronic or other means, duplication of any material in this paper for a fee or for commercial purposes, or modification of the content of the paper are prohibited. 



\title{
Computer aided cytological cancer diagnosis: Cell type classification as a step towards fully automatic cancer diagnostics on cytopathological specimens of serous effusions
}

\author{
Timna E. Schneider ${ }^{a}$, André A. Bell ${ }^{a}$, Dietrich Meyer-Ebrecht ${ }^{a}$, \\ Alfred Böcking ${ }^{b}$ and Til Aach $^{a}$ \\ ${ }^{a}$ Institute of Imaging and Computer Vision, RWTH Aachen University \\ Templergraben 55, 52056 Aachen, Germany \\ Telephone: +49 (241) 80 27860, Fax: +49 (241) 8022200 \\ Email: $\{$ ts,ab,dme,ta\}@lfb.rwth-aachen.de \\ ${ }^{b}$ Institute of Cytopathology, Heinrich-Heine-University Düsseldorf \\ Moorenstr. 5, 40225 Düsseldorf, Germany \\ Telephone: +49 (211) 81 19346, Fax: +49 (211) 8118402 \\ Email: boecking@uni-duesseldorf.de
}

\begin{abstract}
Compared to histopathological methods cancer can be detected earlier, specimens can be obtained easier and with less discomfort for the patient by cytopathological methods. Their downside is the time needed by an expert to find and select the cells to be analyzed on a specimen. To increase the use of cytopathological diagnostics, the cytopathologist has to be supported in this task.

DNA image cytometry $(D N A-I C M)$ is one important cytopathological method that measures the DNA content of cells based on the absorption of light within Feulgen stained cells. The decision whether or not the patient has cancer is based on the histogram of the DNA values. To support the cytopathologist it is desirable to replace manual screening of the specimens by an automatic selection of relevant cells for DNA-ICM. This includes automated acquisition and segmentation of focused cells, a recognition of cell types, and a selection of cells to be measured. As a step towards automated cell type detection we show the discrimination of cell types in serous effusions on a selection of about 3,100 manually classified cells. We present a set of 112 features and the results of feature selection with ranking and a floating-search method combined with different objective functions. The validation of the best feature sets with a k-nearest neighbor and a fuzzy k-nearest neighbor classifier on a disjoint set of cells resulted in classification rates of $96 \%$ for lymphocytes and $96.8 \%$ for the diagnostically relevant cells (mesothelial+ cells), which includes benign and malign mesothelial cells and metastatic cancer cells.
\end{abstract}

Keywords: Methods: feature extraction, classification and classifier design, quantitative image analysis, evaluation and validation, Modalities: microscopic imaging, Diagnostic Task: detection, diagnosis

\section{INTRODUCTION}

To increase the chance of cure and to reduce curative costs it is desirable to detect cancer as early as possible. Cytopathological methods of diagnosis allow to detect a variety of cancers up to three years ahead of conventional histopathological methods ${ }^{1}$ and, moreover, to prevent unnecessary biopsies. ${ }^{2}$ Specimens of cells can be taken non- or minimally invasive with, e.g., tiny brushes or fine needle aspiration biopsies (FNABs). This renders cytopathological methods suitable for preventive diagnostics. One of the main drawbacks of cytopathologic diagnostics is the time needed by an expert for the visual inspection of a specimen under a microscope, searching for malignant or suspicious cells and manually selecting them for a detailed analysis. To overcome the problem of

Further author information: (Send correspondence to Timna E. Schneider)

Timna E. Schneider: E-mail: Timna.Schneider@lfb.rwth-aachen.de, Telephone: +49 (241) 8027860 


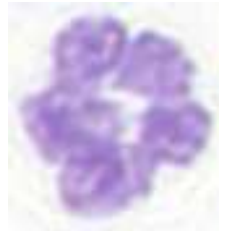

(a)

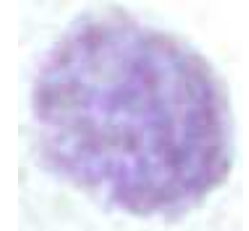

(b)

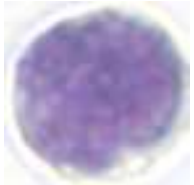

(c)

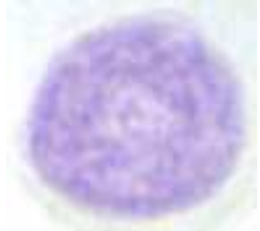

(d)

Figure 1. Different cell types in serous effusions: (a) granulocyte, (b) macrophage, (c) lymphocyte, (d) mesothelial cell.

time needed for a diagnosis, the methods have to be automated such that an expert only has to decide whether or not the selected cells are adequate.

One essential diagnostic method is DNA image cytometry (DNA-ICM) that measures the amount of DNA in cells based on the Feulgen stain, which stains DNA in the nucleus stoichiometrically. Since cancer mostly is a chromosomal abnormality, it thus can be diagnosed from DNA histograms. The process of standardized DNAICM, ${ }^{3}$ based on brightfield microscopical images, is firstly, to manually select about 300 nuclei with abnormal morphology, for which the DNA content should be measured, called analysis cells. Secondly a set of about 30 normal cell nuclei with a DNA value corresponding to a double chromosomal set (2c) has to be selected as reference cells. This set implicates the integral optical density of the stained cells and their DNA content by computing the ratio of the integral optical density of each nucleus to the mean integral optical density of the reference cells. The decision wether or not a patient has cancer is the third and last step. This decision is achieved by evaluating the position of stemlines of the selected population of analysis cells within a histogram, i.e., evaluating number and position of modes of the DNA values and by identifying cells with an abnormal DNA value. Trying to automate this method means to automatically acquire images of nuclei, segment them and select the diagnostically relevant ones, i.e., classify and pre-elect a choice of analysis cells and of reference cells.

Current strategies in automating cytopathological diagnostic methods are limited to a special material, on special stains, and on dedicated special imaging systems, ${ }^{4-8}$ such not. For an automated DNA-ICM a machine is available for screening of cervical smears, using a brightfield light microscope. It searches for so-called exceeding events only, i.e., cells with an abnormal high DNA content, by measuring the DNA value of all existing cells. ${ }^{9}$ But as these cells are rare the same high sensitivity and specificity as with the standardized interactive DNA-ICM, ${ }^{3}$ for which in turn an expert has to select the cells to be measured manually, are not possible. The approach in this paper aims at an algorithmic implementation of such expert knowledge for specimens of serous effusions, i.e., an automatic detection and selection of analysis cells and reference cells in serous effusions.

In these specimens abnormal mesothelial+ cells have to be identified as analysis cells and lymphocytes are the preferred type of reference cells to be sought after. Furthermore these cells have to be distinguished from other cell types occurring in these specimens, e.g., granulocytes and macrophages. Technically speaking a set of mathematical features and a classifier have to be identified to distinguish best between the different types of cells on basis of a manually classified set of cells as a gold standard. Therefore the following sections are organized as follows: In section 2 the different cell types are shown and the imaging modality leading to our large database of cell images are described. On these cell images features have to be computed that characterize properties of the different cell types and that are possibly adequate to distinghuish between them. To decide which subset of features discriminates best between the cell types, a feature selection has to be carried out, followed by training and validating classifiers to achieve best classification results for the selected feature subsets. The algorithms we used for this are explained in section 3. Based on the data set and these algorithms we carried out several experiments, described in section 4, trying to find the combination of algorithms that maximizes the overall classification rate. The results (section 5) of the experiments show, that lymphocytes and mesothelial+ cells can be discriminated at overall classification rates between $95.4 \%$ and $99 \%$. The paper ends up with the conclusions and some future work in section 6 .

\section{MATERIAL}

Our dataset is based on Feulgen stained nuclei from serous effusions with the contours of the nuclei given as chaincodes. An experienced cytopathologist classified 14,346 mesothelial+ cells, 1,915 lymphocytes, and 95 
granulocytes that have been acquired during the DNA-ICM of 58 specimens. The class of the mesothelial+ cells comprises normal, reactive and neoplastic mesothelial cells and metastatic tumor cells.

These specimens include 2 aszites without cancer, due to heart congestion or inflammations and 13 benign mesotheliomas and 14 metastatic carcinomas (including 9 from the lung, 2 from the ovary, 2 from the breast and one of an unknown primary tumor). Further from pleural effusions 19 without cancer, 6 benign mesotheliomas and 4 due to metastatic carcinomas (including 2 from the stomach and 2 from the pancreas).

Some cell types that might occur in serous effusions are shown in figure 1. Image acquisition was performed by a brightfield light microscope and a $63 x$ oil immersion objective and a three-chip CCD camera with a resulting resolution of $\approx 0.1 \mu \mathrm{m}$ for a pixel edge length.

\section{METHODS}

The generation of a supervised classifier to distinguish between cell types can be split into three parts. Part one: Generation of features, i.e., to compute a mathematical set of features, which describes the cells. Part two: Feature selection, i.e., to reduce the computational time needed for a classification of objects and avoid the effect of the peaking phenomenon (degraded performance of a classifier due to a to small number of training samples relative to the the number of features). This means to search for a, depending on the number of training samples, minimal, efficient choice of features to ensure optimal classification results. Part three: Classification and validation, i.e., to train a classifier according to the chosen features and validating this classifier with a sample set that is disjoint to the training set to rate the quality of this classifier.

\subsection{Generation of features}

Cytopathologists distinguishes between different cell types by considering geometrical properties such as size, shape, characteristics of the nuclear contour and texture of the chromatine structure within the nucleus. On the basis of transmitting light microscopy images of Feulgen stained nuclei and their segmentations, we therefore extracted 112 features. This feature collection is motivated by Rodenacker ${ }^{10}$ (Appendices B2 to B4) and supplemented with $\mathrm{Suk}^{11}$ and comprises some basic geometrical features like area, perimeter, form factor, etc. Additionally we use form describing features based on Fourier descriptors, and on affine invariant ${ }^{10}$ as well as independent affine invariant moments ${ }^{11}$ derived from the central twodimensional polynomial moments, that are computed for the nuclear mask. This feature set is supplemented by texture features. These are the affine invariant and the independent affine invariant moments computed for the extinction image, that is based on the green channel of the original RGB image, and the flat texture image, which is the difference between the extinction image and its median filtered version, here using a filter kernel of size five for the median filter.

\subsection{Feature selection}

To generate subsets of these features with a good separability performance without testing all possible subsets we chose two feature selection methods. The first is the ranking method, ${ }^{12}$ that iteratively adds that feature, that separates best in the one-dimensional case. As the second method we use the sequential forward floating selection $(S F F S)$ as a wrapper-method, ${ }^{13}$ that does not need any parameter. In each step that feature is added to the existing set, that maximizes a separability criterion. After adding the new feature, the floating back starts. This means that the quality of each subset is tested, that can be formed by removing one feature, such searching for a new combination of size minus one with a higher quality than the previous set of subset size minus one. This is done recursively as long as a smaller feature subset with a higher quality is computed, using the smallest new best feature set as the starting point for adding new features again.

Without having knowlegde of the distribution of the classes in each feature space, we use three different objective functions to measure the class separability of each feature set. Fisher's criterion, that is based on scatter matrices, ${ }^{14}$ selects good feature sets, if the classes have a normal distribution. Mutual information ${ }^{15}$ uses the likelihood of a feature vector within each class to estimate the density distribution, but a binning of the feature space has to be done first to compute the estimate of the distribution. Without considering the distribution of the data within the feature space we use the k-nearest neighbor $(k N N)$ as the third objective function, computing the classification rates with leave-one-out cross-validation on the training set. The kNN has 
been modified to prevent impasse and, furthermore, to downweight the occurrence of training samples of equal distance and different class memberships.

\subsection{Classification and validation}

For a user, who is not an expert in classification algorithms, a classifier should compute reproducible and comprehensible results, together with a confidence measure for its decisions, that furtheron enables a sorting of the samples according to their conspicuity. These aspects together with the missing knowledge and possibly changing behaviour of the distribution for each new subset of features, led to the use of kNN and Fuzzy-kNN ${ }^{16}$ to distinguish between the different types of cells.

For a $\mathrm{kNN}$ we trained a version that makes its decision as soon as $\mathrm{k}$ neighbors belong to the same class, providing a kind of rating of the reliability of the decision by considering the number of nearest neighbors belonging to the decision class. A Fuzzy-kNN computes a measure of membership to each class. It takes into account the distances of the sample to its nearest neighbors, thus reducing the influence of nearest neighbors that are far away as well as showing less confidence in almost empty areas in the feature space. As there are different possibilities to weight the distances to the nearest neighbors, we trained the Fuzzy-kNN with two different weighting functions, at first, by providing a complete membership of the labeled samples into their own class and nonmembership in all other classes, here denoted as F-kNN-a. Further, we trained it by using their distances to the class mean of that class for which the membership is computed, thus taking into account how typical a cell represents one class, especially in the area between the classes, denoted as F-kNN-b. The weighting function of the F-kNN-b partitions the feature space in areas around each class mean, that contains all points that are closer to this class mean than to any other. The density distribution of the classes therefore should be unimodal with equal variances.

For the training of these variants of the kNN we used leave-one-out cross-validation.

\section{EXPERIMENTS}

Randomly we selected a set of 1,500 mesothelial+ cells and 1,500 lymphocytes and we took all 95 granulocytes available. To compare the influence of the training set size on the classification rate we have generated two different training sets for feature selection and classification, i.e., the set of the above mentioned 1,500 lymphocytes and mesothelial+ cells and based on these sets a randomly chosen subset of 500 mesothelial+ cells and lymhocytes each, taking all granulocytes in both training sets. These two training sets contain all 112 features to compute a combination of a feature subset and a classifier that is suitable for our classification task.

Furthermore, we analyze the classification results obtainable with the Euclidean and the Mahalanobis distance respectively. To reduce the computational cost we took the two trainings sets from above and extracted a formerly tested subset of 33 features, ${ }^{17}$ containing basic geometrical features (area, perimeter, largest inscribable circle, form factor, bending energy) as well as the affine invariant features introduced in Rodenacker. ${ }^{10}$

This leads to six basic experiments, for which the feature selection and classifier generation has to be carried out: 1500 all and 500all for the training sets containing all features, 1500reduced and 500reduced for the subset of 33 features using the Euclidean distance measure, and 1500reduced_mahal and 500reduced_mahal for the experiments using the Mahalanobis distance.

For each of these six experiments we normalized the feature values to the range $[0,1]$ and computed suitable feature subsets using both the ranking and the SFFS method in combination with each objective function (section 3 ). We searched for feature subsets up to feature set sizes of 40 for all features, and up to a set size of 20 for the reduced initial feature set of 33 features. Each combination of feature set selection methods and objective functions has been investigated, using k-nearest neighbor objective functions up to $k=5$.

To obtain a classification rate independent of the training set, we validated the best combination of each experiment with k-nearest neighbor and fuzzy k-nearest neighbor for both DNA-ICM relevant types, mesothelial+ cells and lymphocytes, on basis of a set of 400 samples for both classes, each disjoint to the used training set and up to $k=10$.

As the quality measure for the classifiers we selected the overall classification rate of all classes. 
Table 1. Results of the feature selection for each experiment using the ranking method. Listed are the classification results $(\mathrm{g} ; \mathrm{m} ; \mathrm{l} ; \mathrm{t})$ in percentages for the ( $\mathrm{g}$ )ranulocytes; $(\mathrm{m})$ esothelial cells; (l)ymphocytes, and the (t)otal rate over all classes, additionally the objective function $((\mathrm{m})$ utual information, the (s)catter matrix criterion, or the $(\mathrm{k}) \mathrm{NNx}$ with $\mathrm{x}$ number of neighbors), the number of features as well as the number of neighbors of the kNN that led to the best classifier of each experiment.

\begin{tabular}{llllc}
\hline experiment & $\begin{array}{l}\text { classification } \\
(\mathrm{g} ; \mathrm{m} ; \mathrm{l} ; \mathrm{t})\end{array}$ & $\begin{array}{l}\text { objective } \\
\text { function }\end{array}$ & $\begin{array}{l}\text { number of } \\
\text { features }\end{array}$ & $\begin{array}{l}\text { number of } \\
\text { neighbors }\end{array}$ \\
\hline 1500all & $64.2 ; 97.2 ; 97.8 ; 96.5$ & $\mathrm{k} 1$ & 29 & 5 \\
500all & $83.2 ; 96.2 ; 96.6 ; 95.3$ & $\mathrm{k} 1$ & 4 & 2 \\
1500reduced & $62.1 ; 96.8 ; 98.1 ; 96.3$ & $\mathrm{k} 1$ & 11 & 4 \\
500reduced & $78.9 ; 95.8 ; 97.8 ; 95.3$ & $\mathrm{k} 1$ & 10 & 3 \\
1500reduced_mahal & $76.8 ; 96.6 ; 97.6 ; 96.5$ & $\mathrm{k} 5$ & 6 & 3 \\
500reduced_mahal & $85.3 ; 94.2 ; 97.8 ; 95.1$ & $\mathrm{~m}$ & 9 & 9 \\
\hline
\end{tabular}

Table 2. Results of the feature selection for each experiment using SFFS. Listed are the classification results (g;m;l;t) in percentages for the (g)ranulocytes; (m)esothelial cells; (l)ymphocytes, and the (t)otal rate over all classes, additionally the objective function $((\mathrm{m})$ utual information, the (s)catter matrix criterion, or the $(\mathrm{k}) \mathrm{NNx}$ with $\mathrm{x}$ number of neighbors), the number of features as well as the number of neighbors of the kNN that led to the best classifier of each experiment.

\begin{tabular}{llllc}
\hline experiment & $\begin{array}{l}\text { classification } \\
(\mathrm{g} ; \mathrm{m} ; \mathrm{l} ; \mathrm{t})\end{array}$ & $\begin{array}{l}\text { objective } \\
\text { function }\end{array}$ & $\begin{array}{l}\text { number of } \\
\text { features }\end{array}$ & $\begin{array}{l}\text { number of } \\
\text { neighbors }\end{array}$ \\
\hline 1500all & $84.2 ; 97.0 ; 97.5 ; 96.8$ & $\mathrm{k} 2$ & 12 & 2 \\
500all & $88.4 ; 97.6 ; 97.0 ; 96.5$ & $\mathrm{k} 1$ & 5 & 3 \\
1500reduced & $72.6 ; 96.7 ; 98.1 ; 96.6$ & $\mathrm{k} 4$ & 5 & 6 \\
500reduced & $88.4 ; 95.2 ; 97.0 ; 95.4$ & $\mathrm{k} 5$ & 3 & 4 \\
1500reduced_mahal & $80.0 ; 96.0 ; 98.3 ; 96.6$ & $\mathrm{~m}$ & 7 & 5 \\
500reduced_mahal & $88.4 ; 94.6 ; 97.4 ; 95.3$ & $\mathrm{~m}$ & 4 & 4 \\
\hline
\end{tabular}

\section{RESULTS}

We identified, eparately for the feature selection methods ranking and SFFS, the classifier with the best total classification rate for each experiment, as is shown in table 1 for the ranking method and in table 2 for SFFS. For each experiment this is done by selecting the best classifier among all objective functions, whereas the best classifier of each objective function is identified on the basis of the best total classification rate of a kNN computed for the training data. Comparing both tables, it can be seen that the classification results obtained with feature selection based on SFFS are slightly better than those obtained with the ranking method and that the size of the feature subsets is smaller, thus needing less computation time.

The resulting feature sets selected as the best during the feature selection on basis of the reduced feature set contain mainly the basic geometrical features (see section 3), whereas the features selected over all features mainly comprises a selection of the moment based features.

The classifier with the best classification rate on the training data is achieved for 1500 all using the SFFS method, i.e. using 1500 mesothelial+ cells and lymphocytes each within the training set and searching for suitable feature subsets within the whole set of features (first row in table 2). Nevertheless, the results obtained with the reduced set of features for the feature selection show comparable results.

The classification results for the validation set are shown in table 3 and in table 4 . As can be seen, for $\mathrm{kNN}$ and F-kNN-a the classification results for mesothelial+ cells and lymphocytes differ slightly from the classification results of the trainings set, but, in total, classifying with the $\mathrm{kNN}$ computes the best classification rates.

The comparatively weak results of the F-kNN-b show, that the pre-condition of this classifier is not fulfilled, i.e., this indicates that the two classes have different variances and/or do not only have one mode. 
Table 3. Results of the different $\mathrm{kNN}$ variants for each filter experiment with $k$ of the best classifier (2). Listed are the classification results $(\mathrm{m} ; \mathrm{l} ; \mathrm{t})$ in percentages for the (m)esothelial cells; (l)ymphocytes, and the (t)otal rate over all classes, and additionally the number of features as well as the number of neighbors of the $\mathrm{kNN}$ that led to the best classifier of each experiment.

\begin{tabular}{lllllc}
\hline experiment & $\begin{array}{l}\mathrm{kNN} \\
(\mathrm{m} ; \mathrm{l} ; \mathrm{t})\end{array}$ & $\begin{array}{l}\text { F-kNN-a } \\
(\mathrm{m} ; \mathrm{l} ; \mathrm{t})\end{array}$ & $\begin{array}{l}\text { F-kNN-b } \\
(\mathrm{m} ; \mathrm{l} ; \mathrm{t})\end{array}$ & $\begin{array}{l}\text { number of } \\
\text { features }\end{array}$ & $\begin{array}{l}\text { number of } \\
\text { neighbors }\end{array}$ \\
\hline 1500all & $97.5 ; 97.5 ; 97.5$ & $95.5 ; 98.0 ; 96.8$ & $81.5 ; 96.3 ; 88.9$ & 12 & 2 \\
500all & $94.0 ; 96.8 ; 95.4$ & $93.5 ; 95.8 ; 94.6$ & $77.8 ; 95.5 ; 86.6$ & 5 & 3 \\
1500reduced & $97.5 ; 97.5 ; 97.5$ & $96.5 ; 98.0 ; 96.8$ & $81.5 ; 96.3 ; 88.9$ & 5 & 6 \\
500reduced & $95.5 ; 97.3 ; 99.0$ & $95.5 ; 96.0 ; 95.8$ & $79.3 ; 96.3 ; 87.8$ & 3 & 4 \\
1500reduced_mahal & $97.0 ; 97.0 ; 96.9$ & $91.8 ; 97.3 ; 94.5$ & $77.0 ; 93.0 ; 85.0$ & 7 & 5 \\
500reduced_mahal & $94.0 ; 97.0 ; 95.5$ & $49.3 ; 93.0 ; 71.1$ & $79.5 ; 97.5 ; 88.5$ & 4 & 4 \\
\hline
\end{tabular}

Table 4. Results of the different kNN variants for each SFFS experiment with $k$ of the best classifier (2). Listed are the classification results $(\mathrm{m} ; \mathrm{l} ; \mathrm{t})$ in percentages for the (m)esothelial cells; (l)ymphocytes, and the (t)otal rate over all classes, and additionally the number of features as well as the number of neighbors of the $\mathrm{kNN}$ that led to the best classifier of each experiment.

\begin{tabular}{lllllc}
\hline experiment & $\begin{array}{l}\mathrm{kNN} \\
(\mathrm{m} ; \mathrm{l} ; \mathrm{t})\end{array}$ & $\begin{array}{l}\text { F-kNN-a } \\
(\mathrm{m} ; \mathrm{l} ; \mathrm{t})\end{array}$ & $\begin{array}{l}\text { F-kNN-b } \\
(\mathrm{m} ; \mathrm{l} ; \mathrm{t})\end{array}$ & $\begin{array}{l}\text { number of } \\
\text { features }\end{array}$ & $\begin{array}{l}\text { number of } \\
\text { neighbors }\end{array}$ \\
\hline 1500all & $95.5 ; 96.8 ; 96.1$ & $95.5 ; 96.3 ; 95.9$ & $81.0 ; 99.3 ; 90.1$ & 12 & 2 \\
500all & $96.8 ; 96.0 ; 96.4$ & $96.5 ; 96.0 ; 96.3$ & $77.3 ; 95.0 ; 86.1$ & 5 & 3 \\
1500reduced & $96.5 ; 98.0 ; 97.3$ & $96.5 ; 98.0 ; 97.3$ & $75.8 ; 1.0 ; 87.9$ & 5 & 6 \\
500reduced & $99.3 ; 98.8 ; 99.0$ & $98.0 ; 98.3 ; 98.1$ & $87.3 ; 98.5 ; 92.9$ & 3 & 4 \\
1500reduced_mahal & $95.8 ; 98.0 ; 96.9$ & $87.3 ; 98.8 ; 93.0$ & $73.3 ; 84.5 ; 78.9$ & 7 & 5 \\
500reduced_mahal & $95.3 ; 98.0 ; 96.7$ & $88.8 ; 97.5 ; 93.1$ & $74.5 ; 99.5 ; 87.0$ & 4 & 4 \\
\hline
\end{tabular}

Using the Mahalanobis distance as the distance measure does not always increase the classification rate, as can be seen by comparing the results 1500reduced_mahal with 1500reduced and 500reduced_mahal with 500reduced for both training and validation results. During the feature selection process for the experiments using the Mahalanobis distance, some of the feature subsets could not be rated. For these the inverse of the covariance matrix estimate, needed to compute the Mahalanobis distance, could not be computed, as the covariance matrix estimate was singular.

For both feature selection and classifier validation, comparing the classification results of each experiment between the two data sets of 1500 and 500 cells for lymphocytes and mesothelial+ cells each shows, that the $\mathrm{kNN}$ nearly always provides lower rates for the smaller sets than for the sets of $1500^{*}$, but at the same time often needs a smaller number of nearest neigbors and fewer features. This is not always true for the Fuzzy-kNN classifier (see table 4), which indicates that the density of the training data of both classes around the samples to be classified is high. Therefore the distance weighting of the Fuzzy-kNN variants, which are a kind of is nearer to class $x$, cannot enhance the classification result.

Therefore concentrating on the classification rates of the $\mathrm{kNN}$ for the validation set results in a maximal difference of $5.3 \%$ percentage points over all experiments for the differences of the classification rates of each class as well as for the overall classification rate and in classification rates nearly always above $95 \%$. As an example some classification results of the classifier 1500all of table 2 are shown in figure 2 for the lymphocytes and in figure 3 for the mesothelial+ cells.

As an oversight of relevant cells may result in a false-negative diagnosis, i.e. the cancer will not be detected, one should choose that classifier that includes most analysis cells. Minor missclassifications of reference cells will not affect the diagnostic result, as only the mean value of their optical densities will be taken. Therefore taking 
the classifier with the highest classification rate for the mesothelial+ cells after the training process (500all in table 2) results for the validation cell set in classification rates of $96.8 \%$ for the mesothelial+ cells and $96 \%$ for the lymphocytes. Repeating feature selection and training of the classifiers based on the classification rates of the mesothelial+ cells might enhance the detection rate of these cells, thus reducing the risk of a false-negative diagnosis. But, so far, it can be stated that the current results already provide an encouraging basis to distinguish between lymphocytes and mesothelial+ cells.

\section{CONCLUSIONS AND FUTURE WORK}

The results of F-kNN-b and a classifier using the Mahalanobis distance confirm, that the choice of non-parametric classifiers was the right one. Especially during the process of feature selection, good feature combinations are not necessarily independent, nor normally distributed. Since computing the Mahalanobis distance also increases the time needed for computation, this should not be used during the feature selection, but it is an alternative to possibly enhance the resulting classifier.

Comparing the results of the different experiments led to comparable classification results for different feature sets and different classifiers, thus offering the choice to choose a classifier that is a compromise of the number of nearest neighbors, the number of features, and then to combine it with a rating of the reliability of the decision.

Depending on the application, the strategy to rate the classifiers according to their overall classification result can be modified to choose these classifiers that achieves best classification results for one class. But this may sometimes lead to really worse rates for other classes. So, with our strategy to select the classifiers according to the best overall classification rate we could show, that with the $\mathrm{kNN}$ the obtainable classification rates of each class as well as the overall rate are stable and at a hight rate and comparable for lymphocytes and mesothelial+ cells.

As the next step, the classifiers have to be tested under routine conditions, i.e., try to identify lymphocytes and mesothelial+ cells among automatically segmented objects. This might possibly lead to the necessity of collecting prototypes of granulocytes, and macrophages, as well as artefact segmentations or lytic cells. Furthermore, within the set of mesothelial+ cells a rating has to be introduced to provide the cytopathologist with a pre-election of cells being most suspicious of cancer.

However, overall we could show that it is possible to distinguish mesothelial+ cells and lymphocytes within a manually classified set of cells from serous effusions at a rate of $96.8 \%$ and $96 \%$ respectively. As these are the relevant classes for the DNA-ICM of serous effusion, this provides a promising approach to distinguish these cells within automatically segmented objects of a whole specimen. This way an automation of DNA-ICM for serous effusion becomes feasible.

\section{ACKNOWLEDGMENTS}

The project is supported by the Viktor-and-Mirka-Pollak-Fund for Biomedical Engineering. 


\begin{tabular}{|c|c|c|c|}
\hline $\begin{array}{l}\text { lymphocyte from } \\
\text { the validation set }\end{array}$ & $\begin{array}{l}\text { first nearest } \\
\text { neighbor }\end{array}$ & $\begin{array}{l}\text { second nearest } \\
\text { neighbor }\end{array}$ & $\begin{array}{l}\text { third nearest } \\
\text { neighbor }\end{array}$ \\
\hline $\begin{array}{c}\text { lymphocyte falsely classified } \\
\text { as mesothelial+ cell }\end{array}$ & mesothelial+ cell & mesothelial + cell & \\
\hline $\begin{array}{l}\text { lymphocyte falsely classified } \\
\text { as mesothelial+ cell }\end{array}$ & mesothelial+ cell & lymphocyte & mesothelial+ cell+ \\
\hline $\begin{array}{c}\text { lymphocyte falsely classified } \\
\text { as mesothelial+ cell }\end{array}$ & lymphocyte & mesothelial+ cell & mesothelial + cell \\
\hline classified as lymphocyte & mesothelial + cell & lymphocyte & lymphocyte \\
\hline classified as lymphocyte & lymphocyte & mesothelial + cell & lymphocyte \\
\hline classified as lymphocyte & lymphocyte & lymphocyte & \\
\hline
\end{tabular}

Figure 2. Classification results for lymphocytes. The first column contains lymphocytes of the validation set that were classified with the 500all classifier from table 2. The nearest neighbors that caused the decisions are shown in columns two to three, sorted from left to right in increasing distance in feature space to the classified cell in column one. 


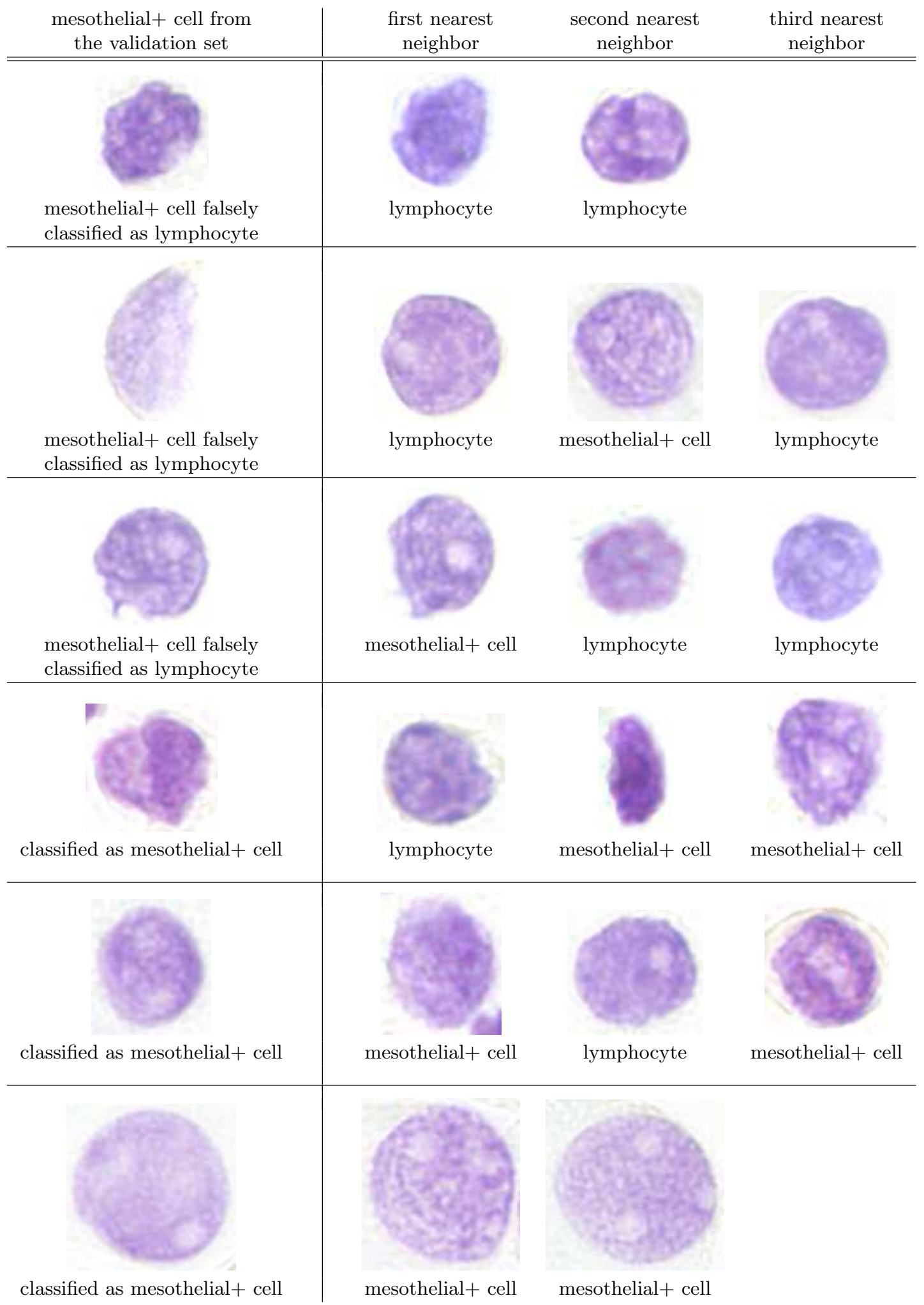

Figure 3. Classification results for mesothelial+ cell. The first column contains mesothelial+ cell of the validation set that were classified with the 500all classifier from table 2 . The nearest neighbors that caused the decisions are shown in columns two to three, sorted from left to right in increasing distance in feature space to the classified cell in column one. 


\section{REFERENCES}

1. T. Remmerbach, H. Weidenbach, N. Pomjanski, K. Knops, S. Mathes, A. Hemprich, and A. Böcking, "Cytologic and DNA-cytometric early diagnosis of oral cancer," Anal Cell Pathol 22(4), pp. 211-221, 2001.

2. A. Carpi, E. Ferrari, M. Toni, A. Agripanti, A. Nicolini, and G. D. Coscio, "Needle aspiration techniques in preoperative selection of patients with thyroid nodules: a long-term study." J Clin Oncol 14(5), pp. 1704$1712,1996$.

3. A. Böcking and et al., "Consensus report of the ESACP task force on standardization of diagnostic DNA image cytometry," Analytical Cellular Pathology 8(1), pp. 67-74, 1995.

4. F. Scotti, "Automatic morphological analysis for acute leukemia identification in peripheral blood microscope images," IEEE Computational Intelligence for Measurement Systems and Applications , pp. 96-101, 2005.

5. Q. Wu, L. Zeng, H. Ke, W. Xie, H. Zheng, and Y. Zhang, "Analysis of blood and bone marrow smears using multispectral imaging analysis techniques," Proc. SPIE on Medical Imaging 5747, pp. 1872-1882, 2005.

6. Z.-H. Zhou, Y. Jiang, Y.-B. Yang, and S.-F. Chen, "Lung cancer cell identification based on artificial neural network ensembles," Artificial Ingelligence in Medicine 24, pp. 25-36, 2002.

7. S. McKenna, I. Ricketts, A. Cairns, and K. Hussein, "A comparison of neural network architectures for cervical cell classification," Proc. Third International Conference on Artificial Neural Networks , pp. 105109, 1993.

8. M. Olivo, W. Lau, V. Manivasager, R. Bhuvaneswari, and Z. Wei, "Novel photodynamic diagnosis of bladder cancer: Ex vivo fluorescence cytology using hypericin," International Journal Of Oncology 23, pp. 1501$1504,2003$.

9. X. Sun, J. Wanq, D. Garner, and B. Palcic, "Detection of cervical cancer and high grade neoplastic lesions by a combination of liquid-based sampling preparation and dna measurements using automated image cytometry," Cellular Oncology 27(8), pp. 33-41, 2005.

10. K. Rodenacker and E. Bengtsson, "A feature set for cytometry on digitized microscopic images," Analytical Cellular Pathology 25(1), pp. 1157-1182, 2003.

11. T. Suk and J. Flusser, "Graph method for generating affine moment invariants," in 17th International Conference on Pattern Recognition (ICPR'04), 2, pp. 192-195, 2004.

12. I. Guyon and A. Elisseeff, "An introduction to variable and feature selection," Journal of Machine Learning Research 3, pp. 1157-1182, 2003.

13. P. Pudil, J. Novovicova, and J. Kittler, "Floating search methods in feature selection.," Pattern Recognition Letters (15), pp. 1119-1125, 1994.

14. K. Fukunaga, Statistical Pattern Recognition, Academic Press, 2 ed., 1990.

15. J. Pluim, J. Maintz, and M. A. Viergever, "Mutual information based registration of medical images: a survey," IEEE Transactions on Medical Imaging 22(8), pp. 986-1004, 2003.

16. J. Keller, M. Gray, and J. G. Jr, "A fuzzy k-nearest neighbor algorithm," IEEE Transactions on Systems, Man, and Cybernetics (4), pp. 580-585, 1985.

17. M. Zarzycki, T. Schneider, D. Meyer-Ebrecht, and A. Böcking, "Classification of cell types in Feulgen stained cytologic specimens using morphologic features," in Bildverarbeitung für die Medizin. BVM 2005, pp. 410-414, 2005. 